\title{
COVID-19: persistence of symptoms and lung alterations after 3-6 months from hospital discharge
}

\author{
Alberto Fortini ${ }^{1}$ (I) A Arianna Torrigiani ${ }^{1} \cdot$ Serena Sbaragli ${ }^{1} \cdot$ Aldo Lo Forte $^{1} \cdot$ Andrea Crociani $^{1} \cdot$ Paolo Cecchini $^{2}$. \\ Giulia Innocenti Bruni ${ }^{3}$. Antonio Faraone ${ }^{1}$
}

Received: 12 January 2021 / Accepted: 31 May 2021 / Published online: 6 June 2021

○) Springer-Verlag GmbH Germany, part of Springer Nature 2021

\begin{abstract}
Purpose Few data are currently available on persistent symptoms and late organ damage in patients who have suffered from COVID-19. This prospective study aimed to evaluate the results of a follow-up program for patients discharged from a nonintensive COVID-19 ward.

Methods 3-6 months after hospital discharge, 59 of 105 COVID-19 patients (31 males, aged 68.2 \pm 12.8 years) were recruited in the study. Forty-six patients were excluded because of nontraceability, refusal, or inability to provide informed consent. The follow-up consisted of anamnesis (including a structured questionnaire), physical examination, blood tests, ECG, lower limb compression venous ultrasound (US), thoracic US, and spirometry with diffusion lung capacity for carbon monoxide (DLCO). Results $22 \%$ of patients reported no residual symptoms, $28.8 \% 1$ or 2 symptoms and $49.2 \% 3$ or more symptoms. The most frequently symptoms were fatigue, exertional dyspnea, insomnia, and anxiety. Among the inflammatory and coagulation parameters, only the median value of fibrinogen was slightly above normal. A deep vein thrombosis was detected in 1 patient (1.7\%). Thoracic US detected mild pulmonary changes in 15 patients $(25.4 \%), 10$ of which reported exertional dyspnea. DLCO was mildly or moderately reduced in 19 patients (37.2\%), 13 of which complained of exertional dyspnea.

Conclusion These results highlight that a substantial percentage of COVID-19 patients (77.8\%) continue to complain of symptoms 3-6 months after hospital discharge. Exertional dyspnea was significantly associated with the persistence of lung US abnormalities and diffusing capacity alterations. Extended follow-up is required to assess the long-term evolution of postacute sequelae of COVID-19.
\end{abstract}

Keywords COVID-19 $\cdot$ Follow-up $\cdot$ DLCO $\cdot$ Spirometry $\cdot$ Lung ultrasound

\section{Introduction}

Coronavirus disease 2019 (COVID-19) has a variable spectrum of clinical manifestations: most patients develop mild to moderate disease with a favorable prognosis, while about $20 \%$ manifest a serious or even critical illness [1-4]. In the latter patients, acute respiratory failure, resulting from the development of bilateral interstitial pneumonia, is mostly

Alberto Fortini

xfortini@gmail.com; alberto.fortini@uslcentro.toscana.it

1 Internal Medicine, San Giovanni di Dio Hospital, Via di Torregalli 3, 50143 Florence, Italy

2 Emergency Department, San Giovanni di Dio Hospital, Florence, Italy

3 San Giovanni di Dio Hospital, Florence, Italy observed. However, multisystemic complications are also possible due to direct damage by the virus and/or to the hyperinflammatory and procoagulative cytokine-mediated host response [5]. Although the clinical picture of the acute phase has been well described, less is known about the clinical evolution of COVID-19 patients after hospital discharge. At present, the available data from follow-up studies are limited. Preliminary analyses indicate that a substantial subset patients continue to complain of persistent symptoms after several months [6-10]. In some cases, organ damage is found, especially in the lungs [10-12]. To identify and manage the postacute sequelae of COVID-19 still present 3-6 months after hospital discharge, we implemented an outpatient post-COVID-19 follow-up program. The purpose of the current prospective study was to evaluate the results of this post-COVID-19 follow-up program to shed light on the 
long-term consequence of COVID-19 and plan the appropriate interventions.

\section{Methods}

\section{Study design and participants}

A single-center prospective study of patients with a previous diagnosis of SARS-CoV-2 infection was conducted in the San Giovanni di Dio Hospital in Florence, Italy. All patients discharged from the nonintensive COVID-19 ward during the first wave of the pandemic (from March to May 2020) were considered for inclusion in the study. They were invited by phone call to participate in a structured post-COVID-19 follow-up program, to be performed 3-6 months after hospital discharge. The exclusion criteria were: (a) non traceability; (b) refusal or inability to provide informed consent; c) inability to carry out the follow-up program for clinical or logistical reasons.

\section{Follow-up program}

The follow-up program was developed and carried out by a team of internal medicine physicians with the collaboration of two pulmonologists, according to the recommendation of the Tuscany region for the management of patients affected by COVID-19 (Tuscany region resolution $n^{\circ} 744$, June 6 , 2020).

The patients were scheduled for two consecutive ambulatory visits two weeks apart. Two days before the first visit, they underwent a nasopharyngeal swab for the search for SARS-CoV-2 viral RNA, using a real-time reverse transcriptase-polymerase chain reaction. At the first visit, the patients were asked to provide written informed consent to participate in the study. Subsequently, they completed a 46-item self-administered questionnaire designed by the Health Service of the Tuscany Region, aiming to assess the persistence of symptoms complained of during hospitalization. Then, they underwent a comprehensive panel of laboratory and instrumental tests (Table 1), to investigate the persistence of inflammatory and coagulative changes, venous thromboembolic complications, and respiratory system disorders.

Thoracic ultrasound (US) was used to assess the presence of residual lung abnormalities. The sonographic examination was performed according to the protocol described by Bosso et al. A convex or linear array probes were used to explore 12 wall zones, 6 per side, with the patient in a sitting position [13]. Each zone was scored according to the lung US pattern (score 0: presence of A-lines or fewer than 3 isolated B-lines, score 1: presence of multiple well-spaced B-lines, score 2: presence of coalescent B-lines, score 3: presence of lung consolidation). The final score (range 0-36) was obtained by summing up the scores of each area. The extent of pulmonary involvement was categorized as mild (score 1-12), moderate (score 13-24), or severe (25-36).

The presence of deep vein thrombosis (DVT) of the lower extremities was assessed through compression ultrasonography (CUS). The common femoral vein, the femoral vein and the popliteal vein (3-point CUS) were explored bilaterally with a $7.5 \mathrm{MHz}$ linear array probe in B-mode, using a transverse compression technique [14]. DVT was diagnosed by the inability to fully compress the explored veins.

Thoracic US and CUS were performed by internal medicine physicians during the follow-up visit.

The respiratory evaluation was performed by pulmonologists, using spirometry, and blood gas analysis. The spirometric parameters were: forced vital capacity (FVC), forced expiratory volume in one second (FEV1), total lung capacity

Table 1 Post-COVID-19 follow-up program

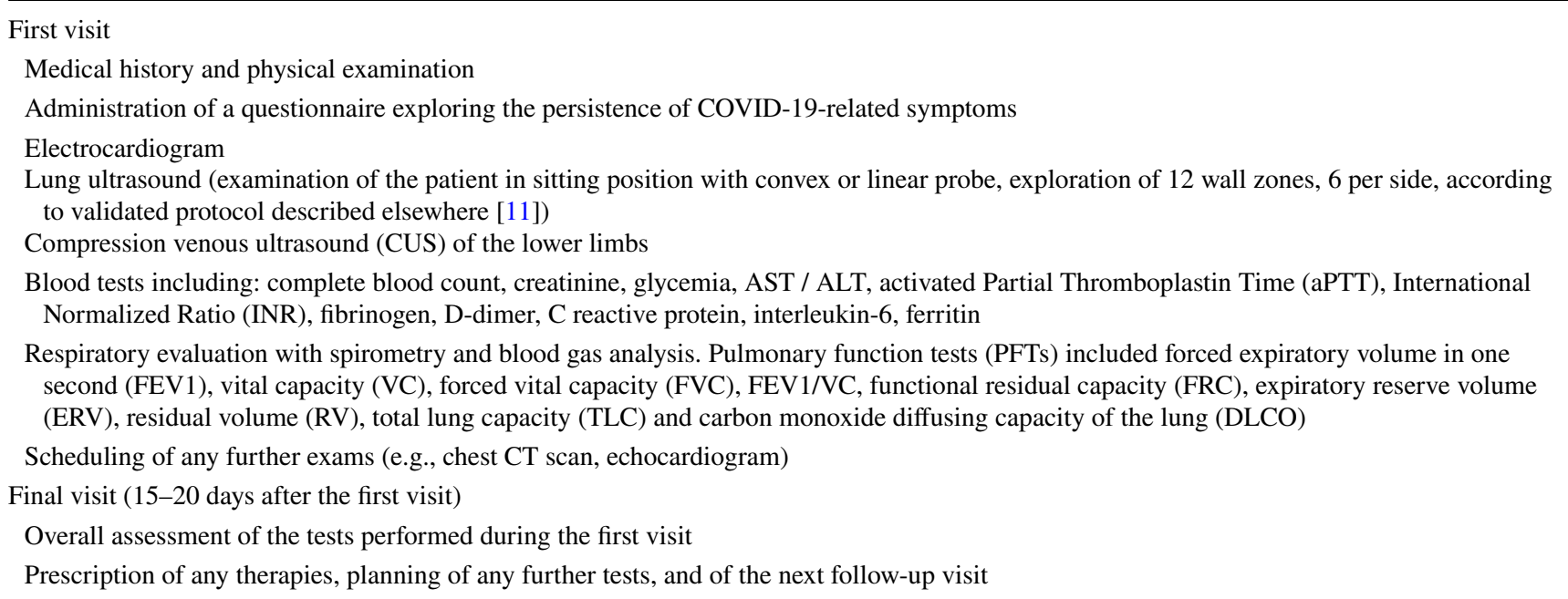


(TLC), and carbon monoxide diffusing capacity of the lung (DLCO).

At a second visit, the internal medicine physician assessed the results of the performed tests and planned any further therapies and diagnostic tests. All patients were then scheduled for a conclusive visit 12 months after the hospital discharge.

\section{Objectives of the study}

The objectives of the study were to assess:

- the persistence of symptoms related to COVID-19;

- the persistence of anomalies in blood tests; namely, in coagulation and inflammatory parameters;

- the presence of DVT;

- the respiratory function and the lung US morphological characteristics.

\section{Data collection}

The data were collected in a Microsoft Excel spreadsheet (version 2013) by a physician specializing in internal medicine, supervised by two experienced physicians responsible for the COVID-19 ward Unit. The data were de-identified to preserve the anonymity of the participants.

All procedures were performed in accordance with the 1964 Helsinki declaration and its later amendments. The study was approved by the Institutional Review Board of the Department of Medicine of the "Azienda USL Toscana Centro", Firenze, Italy.

\section{Statistical analysis}

GNU PSPP Statistical Analysis Software (https://www.gnu. org/software/pspp/) was used for statistical analyses. The data were presented as number and/or percentage for categorical variables and mean and standard deviation (SD) or median and interquantile range (IQR) when appropriate for continuous ones. The normality of data distribution was assessed using the Shapiro-Wilk test. Normally distributed continuous variables were compared with Student $t$ test, non-normally distributed variables with Mann-Whitney test. Categorical variables were compared with $\chi^{2}$ or Fisher exact test. Multivariable logistic regression model was performed to identify the predictors of symptom persistence. $P<0.05$ was considered statistically significant.

\section{Results}

During the study period, from July 2020 to October 2020, 105 patients were contacted by phone; 46 patients were excluded because of nontraceability, refusal, or inability to provide informed consent.

Fifty-nine patients were included in the study and evaluated after a median of 123 days (interquartile range 116; 145; minimum 98, maximum 195) from discharge. The mean (SD) age was $68.2(12.8)$ years; $31(52.5 \%)$ were male. Patients had a mean of $1.7 \pm 1.6$ comorbidities. The most frequent ones were hypertension (47.5\%), cardiovascular disease (30.1\%), and obesity (16.9\%). The median hospital stay was 12 (IQR, 7-19.5) days. Sixteen patients (27.1\%) received noninvasive ventilation. Baseline characteristics of patients and their comorbidities are described in Table 2.

Six patients $(10.2 \%)$ were re-admitted to the hospital before the follow-up visit for the following reasons: 3 for pneumonia, 2 for respiratory failure, and 1 for rectal bleeding.

At the time of the evaluation, $22 \%$ of patients reported a complete lack of symptoms, while $28.8 \%$ reported the persistence of one or two symptoms and $49.2 \%$ of three or more symptoms. The most frequent symptom was fatigue (42.4\%), followed by dyspnea on exertion (37.3\%). Insomnia (28.8\%),

Table 2 Baseline characteristics and comorbidities of the patients

\begin{tabular}{lc}
\hline & Patients $(n=59)$ \\
\hline Male gender, $N(\%)$ & $31(52.5)$ \\
Age, years, mean (SD) & $68.2(12.8)$ \\
Number of comorbidities, mean (SD) & $1.7(1.6)$ \\
Comorbidities, $N(\%)$ & \\
Hypertension & $28(47.5)$ \\
Cardiovascular disease & $18(30.1)$ \\
Cognitive impairment & $1(1.7)$ \\
Diabetes mellitus & $9(15.2)$ \\
Chronic kidney disease & $2(3.4)$ \\
Chronic obstructive pulmonary disease & $5(8.5)$ \\
Atrial fibrillation & $8(13.6)$ \\
Active cancer & $3(5.1)$ \\
Tobacco exposure & $9(15)$ \\
Stroke & $4(6.8)$ \\
Obesity & $10(16.9)$ \\
Immune disorders & $0(0)$ \\
Liver disease & $1(1.7)$ \\
Noninvasive ventilation, $N(\%)$ & $16(27.1)$ \\
Length of hospital stay, days, median (IQR) & $12(7,19.5)$ \\
Time from discharge to follow-up, days, median & $123(116,145)$ \\
(IQR) & \\
\hline
\end{tabular}

IQR interquartile range 
anxiety, and depression (22\%), were also quite common. Finally, $16.9 \%$ and $15.2 \%$ of the population reported the persistence of alterations in taste and smell, respectively. The reported symptoms are detailed in Fig. 1. According to multivariable logistic regression analysis, age, gender, burden of comorbidity (expressed by the Charlson Comorbidity Index), presence of Chronic Obstructive Pulmonary Disease (COPD), and clinical severity of COVID-19 during hospitalization did not predict the persistence of the most common symptoms complained by patients (fatigue, dyspnea, anxiety, insomnia, depression, ageusia, anosmia).

At the time of follow-up, the mean or median values of laboratory tests were all in the normal range, except for fibrinogen that was slightly increased (Table 3). Taking into account individual patients' D-dimer, fibrinogen, and IL-6 values, they were above the upper limit of the reference range in $32.2 \%, 61 \%$, and $32.2 \%$ of cases, respectively (Table 3). No significant correlation between the values of these parameters and the patient-reported symptoms was detected by the multivariable logistic regression analysis. Compared with the hospital admission, the values of the main inflammation parameters (PCR, IL-6, ferritin) and of D-dimer were all significantly reduced at the follow-up visit (Table 4).

During the acute phase of Covid-19, 53 out of the 59 enrolled patients $(88.9 \%)$ had had lung alterations detected

Fig. 1 Persistent symptoms at the time of follow-up visit

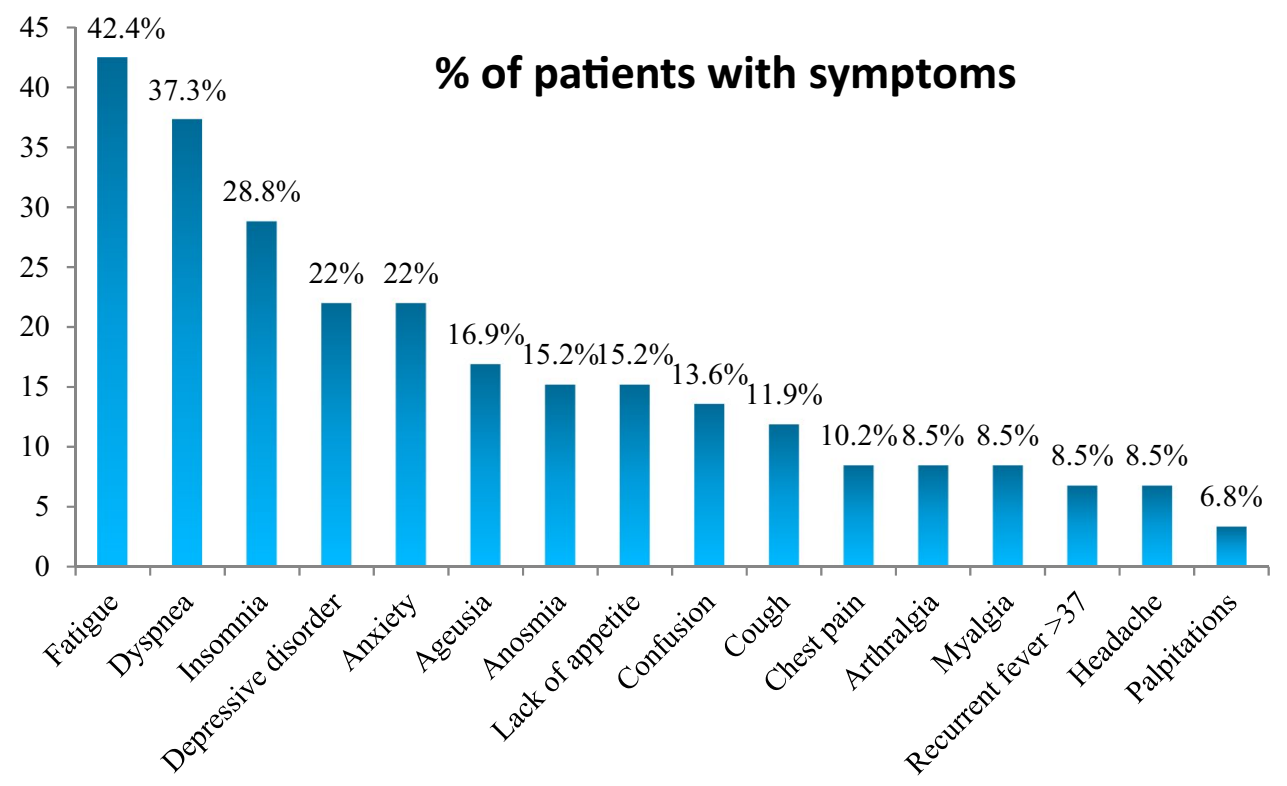

Table 3 Laboratory parameters at the time of follow-up

\begin{tabular}{lccc}
\hline & Value at follow-up & Reference range & $\begin{array}{l}\text { Number of patients }(\%) \text { with } \\
\text { values above the reference } \\
\text { range }\end{array}$ \\
\hline Hemoglobin, g/dl, mean (SD) & & $6(10.2)$ \\
Platelets, $n$./ mmc, mean (SD) & $13.6(1.5)$ & $12-16$ & $3(5.1)$ \\
White blood cells, $n$.mmc, mean (SD) & $213.4(60)$ & $140-450$ & $3(5.1)$ \\
D-dimer, nanogr/ml, median (IQR) & $6484(2092)$ & $4200-5400$ & $19(32.2)$ \\
INR, mean (SD) & $387.5(250 ; 568)$ & $<500$ & $6(10.2)$ \\
aPTT, sec, mean (SD) & $1.1(0.15)$ & $<1.2$ & $2(3.4)$ \\
Fibrinogen, mg/dl, median (IQR) & $31(5.7)$ & $25-37$ & $36(61)$ \\
Creatinine, mg/dl, mean (SD) & $419(349,486)$ & $200-400$ & $13(22)$ \\
Ferritin, ng/ml, median (IQR) & $0.8(0.3)$ & $0.44-0.95$ & $3(5)$ \\
CRP, mg/dl, median (IQR) & $61.5(29.2,92)$ & $20-200$ & $6(10.2)$ \\
IL-6, pg/ml, median (IQR) & $0.2(0.1,0.3)$ & $<0.5$ & $19(32.2)$ \\
NT-proBNP, pg/ml, median (IQR) & $1.5(1.5,3.3)$ & $<3$ & $24(40.1)$ \\
\hline
\end{tabular}

$S D$ standard deviation, $I Q R$ interquartile range, $I N R$ International Normalized Ratio, $a P T T$ activated partial thromboplastin time, $C R P$ C-reactive protein, $I L-6$ interleukin 6, NT-proBNP N-terminal Pro B type natriuretic peptide 
Table 4 Inflammation parameters and D-dimer at the time of hospital admission and at follow-up

\begin{tabular}{lcccl}
\hline & Hospital admission & Follow $=$ up & $p$ & Reference range \\
\hline D-dimer, nanogr/ml, median (IQR) & $902(760,1282)$ & $387.5(250,568)$ & $<0.001<500$ \\
CRP, mg/dl, median (IQR) & $10.8(3.9,16.8)$ & $0.2(0.1,0.3)$ & $<0.001<0.5$ \\
IL-6, pg/ml, median (IQR) & $28.3(11,42.9)$ & $1.5(1.5,3.3)$ & $<0.001<3$ \\
Ferritin, ng/ml, median (IQR) & $466(245,869)$ & $61.5(29.2,92)$ & $<0.001$ & $20-200$
\end{tabular}

$C R P$ C-reactive protein, $I L-6$ interleukin 6
Table 5 Results of arterial blood gas analysis and spirometry with DLCO

\begin{tabular}{lc}
\hline & Patients $(n=51)$ \\
\hline Arterial blood gas & \\
$\mathrm{PaO}_{2} / \mathrm{FiO}_{2}$, mean (SD) & $444(38)$ \\
$\mathrm{Pa}_{2} / \mathrm{FiO}_{2}<350, n .(\%)$ & $2(3.9)$ \\
Spirometry & \\
FVC\% of predicted, mean (SD) & $97.3(14.3)$ \\
FVC $<80 \%$, n. $(\%)$ & $4(7.8)$ \\
FEV1\% of predicted, mean (SD) & $97.3(15.3)$ \\
FEV1 $<80 \% n .(\%)$ & $6(11.8)$ \\
FEV1/FVC $\times 100$, mean (SD) & $98(13.4)$ \\
FEV1/FVC $<70 \% n .(\%)$ & $6(11.8)$ \\
TLC, $\%$ of predicted, mean (SD) & $97.5(21.8)$ \\
TLC $<80 \% n .(\%)$ & $7(13.7 \%)$ \\
DLCO, $\%$ of predicted, mean (SD) & $83.1(20.6)$ \\
DLCO $<80 \%, ~ n .(\%)$ & $19(37.2)$ \\
\hline
\end{tabular}

$\mathrm{PaO}_{2}$ partial pressure of oxygen, $\mathrm{FiO}_{2}$ fraction of inspired oxygen, $F V C$ forced vital capacity, $F E V_{l}$ forced expiratory volume in one second, $V C$ vital capacity, $T L C$ total lung capacity, $D L C O$ carbon monoxide diffusing capacity of the lung

by sonography. Thoracic US at follow-up revealed residual mild lung changes (multiple well-spaced B-lines-score $1-12)$ in 15 of these 53 patients (28.3\%).

NIV during hospitalization was more frequent in patients with residual US abnormalities at follow-up (8/15, 53.3\%) than in patients without residual US abnormalities ( $9 / 44$, $20.5 \% ; P<0.05)$.

CUS of the lower limbs showed a popliteal deep vein thrombosis in one patient (1.7\%).

Pulmonary function tests were performed in 57 patients. Six patients failed to complete the spirometric procedure due to lack of cooperation $(n .=2)$, inability to correctly perform the procedure $(n .=3)$, or presence of uncontrollable cough $(n .=1)$. The analyzed data for the residual 51 patients are shown in Table 5. The mean (SD) $\mathrm{PaO}_{2} /$ $\mathrm{FiO}_{2}$ ratio was 444 (38.2), with any value, except those of 2 patients, greater than 350 . We observed a reduction in TLC, $\mathrm{FVC}$, and FEV1 in $13.7 \%, 7.8 \%$, and $11.8 \%$ of the patients, respectively. Nineteen individuals $(37.2 \%)$ had abnormal DLCO. Of these patients, $1(1.9 \%)$ had a reduction to the lower limit of normal range (DLCO 80-76\%), 13 (25.5\%) a mild reduction (DLCO 61-75\%), and 5 (9.8\%) a moderate reduction (DLCO $41-60 \%$ ). Of the 19 patients with decreased DLCO, 13 (68.4\%) reported persistence of dyspnea, while among 32 patients with normal DLCO, dyspnea was reported in $7(21.9 \%)$ cases $(P<0.01)$. Residual lung US abnormalities were found in 13 of $19(68.4 \%)$ patients with reduced DLCO as compared to 1 of $32(3.1 \%)$ patients with normal DLCO $(P<0.001)$.

Medical history revealed pre-existing COPD in 5 patients. No significant correlation was found between the presence of COPD and pulmonary abnormalities at follow-up.

\section{Discussion}

In this study, we evaluated 59 patients 3-6 months after discharge from the nonintensive COVID-19 ward of our hospital. The main findings were: (i) persistence of one or more symptoms in over 75 per cent of patients; ii) detection of functional and morphological changes in the lungs in over 30 percent of patients. Inflammatory and coagulation parameters were found to be within the normal range in most, although not all, patients, and venous thromboembolic events were extremely rare.

\section{Persistent symptoms}

Most patients still had persistent symptoms up to 180 days after hospital discharge, while only 22 per cent of patients were completely asymptomatic. The most common symptoms were fatigue (42.4\%) and dyspnea (37.3\%). Similar data were recently reported by four studies evaluating COVID-19 patients 60 [6, 7], 90 [8], and 110 days [9] after hospital discharge and by a large Chinese study performed 6 months after hospital discharge [10]. Our data suggest that COVID-19 has a significant impact on patient mental well-being. Namely, in addition to the onset of postdischarge insomnia, about one-fifth of the patients reported anxiety and depression. This finding is in keeping with the previous studies, which reported new-onset psychiatric disorders, such as anxiety, depression, and post-traumatic stress disorder with widely varying prevalence rates [10, 15-17].

Ageusia and anosmia are frequent at the onset of the disease and usually subside within 4 weeks [18]. However, 
some studies have shown that in a minority of patients they can persist beyond a month [19, 20]. In our series, 15.2 and 16.9 per cent of patients, respectively, reported a persistent decrease in senses of smell and taste after hospital discharge. The pathogenetic mechanisms of these chemosensory alterations are still under investigation [18].

Weakness and neuropsychiatric complications, including cognitive impairment, depression, and anxiety have been frequently described in intensive care survivors, and may persist for five or more years [21-23]. It is still debated whether long-time persistence of symptoms in patients hospitalized for COVID-19 is pathogenically similar to that observed in ICU patients admitted for other diseases or is specifically related to SARS-CoV-2 infection. The latter hypothesis is supported by the high prevalence of persistent symptoms even in outpatients with nonsevere COVID-19 [24, 25].

Overall, the occurrence of persistent symptoms after acute SARS-CoV-2 infection is termed "Long Covid" [26], which includes both ongoing symptomatic COVID-19 (signs and symptoms lasting from 4 to 12 weeks) and postCOVID-19 syndrome (signs and symptoms lasting more than 12 weeks).

In our study, the frequency of re-admissions before the follow-up visit was 10.2 per cent, mainly for pneumonia, and/or respiratory failure. Similar re-hospitalization rates have been described by other researchers [6, 7].

\section{Inflammatory and coagulative parameters}

In the acute phase of COVID-19, the presence of a thromboinflammatory state, characterized by high levels of inflammatory parameters, such as CRP, ferritin and Interleukin-6, and some coagulation indices, such as fibrinogen and D-dimer, has been described [27, 28]. These changes were associated with a high frequency of venous thromboembolic complications. Our results show a trend to normalization of these parameters after 3-6 months from hospital discharge. However, a slight, persistent alteration of D-dimer, fibrinogen, and IL-6 was still present in a non-negligible percentage of patients $(32.2 \%, 61 \%$, and $32.2 \%$, respectively). The clinical relevance of these data is not clear. We can hypothesize that in some patients the inflammatory and hypercoagulative state is not completely resolved. However, only a minority of patients complained of persistent fever and other symptoms related to a remarkable inflammatory state (myalgia, arthralgia, headache). Furthermore, although no patient received thromboembolic prophylaxis at the time of evaluation, a venous thromboembolic complication was detected in only 1 patient. Similar data were reported from a French study conducted on over 100 patients to evaluate the risk of venous thromboembolism 2 months after discharge [29]. Although acute COVID-19 patients have a high incidence of thromboembolic complications, the present data suggest that this risk quickly decreases during the convalescence phase.

\section{Thoracic US examination}

Signs of mild interstitial damage (multiple well-spaced B-lines on thoracic ultrasound) were observed in 25.4 per cent of patients. Lung sonographic changes at follow-up were significantly associated with the use of NIV during the acute phase of Covid-19, while they were more rarely found in patients receiving conventional oxygen therapy. Therefore, the persistence of lung sonographic abnormalities appears to be associated with the disease severity during the acute phase.

Thoracic US is a highly sensitive real-time imaging technique, easily performed bedside by skilled clinicians. The results of thoracic US fairly correlate with those of chest CT, and several authors have shown that thoracic US is an accurate noninvasive tool for diagnosis, assessment of disease severity, and monitoring of Covid-19 patients [30, 31]. Our findings appear consistent with previous studies showing the persistence of lung abnormalities in a high percentage of Covid-19 patients undergoing postacute follow-up CT chest imaging $[10,32]$. The data on the performance of thoracic US in the postacute phase of Covid-19 are scant. However, Zhang et al. found pulmonary changes in all 10 patients undergoing thoracic US examination over 30 days after the onset of Covid-19 symptoms [33]. Ultrasonography showed B-lines and thickened pleural line in all patients, and pulmonary consolidation in 7 of them. The sensitivity of the exam for lung abnormalities was comparable to that of chest CT.

\section{Pneumological evaluation}

Almost all patients showed normal arterial oxygenation values, as evidenced by a $\mathrm{PaO}_{2} / \mathrm{FiO}_{2}$ ratio $>350$. However, frequent alterations were found in spirometric parameters. Namely, DLCO was reduced to below $80 \%$ of the predicted value in 37.2 per cent of patients. Although the severity of DLCO reduction was mild (27.4\%) or moderate (9.8\%) in most cases, it was significantly associated with the persistence of dyspnea and signs of interstitial damage on lung ultrasound. The alterations in DLCO reflect a reduction in the diffusion of gases between alveoli and pulmonary capillaries which could be due to damage to the alveolar-capillary membrane. The other spirometric variables under examination (FEV1, FVC, and TLC) were altered in fewer cases $(7.8 \%, 11.8 \%$, and $13.7 \%$, respectively). As our patients did not undergo spirometric evaluation during hospitalization, it was not possible to make a comparison to understand the evolution of lung damage. However, a recent study conducted at the discharge of COVID-19 patients shows a rate and quality of alterations only slightly higher than those we have observed. In particular, 
the authors found a reduction in DLCO in 47.2 per cent of patients, in TLC, FEV1 and FVC in 25, 13, and 9 per cent, respectively [11]. These data suggest that pulmonary changes in the acute phase tend to regress slowly and to be present after a few months from onset. This highlights the need for longterm follow-up and rehabilitation programs.

Our results are consistent with those of previous studies on patients with other coronavirus pneumonia (SARS, MERS), reporting persistent radiological changes, pulmonary function abnormalities, reduced exercise tolerance, and neuropsychiatric symptoms in a sizeable proportion of patients, even beyond 6 months after hospital discharge [34-37]. Hui et al. showed that, 6 months after disease onset, 30 per cent of subjects with the recent diagnosis of SARS had abnormal chest X-Ray, and 4, 7, and 16 per cent of patients had FVC, TLC, and DLCO values below $80 \%$ of predicted, respectively [36]. A recent meta-analysis of 28 long-term complication studies in SARS and MERS survivors showed impaired DLCO (prevalence 27\%) and reduced exercise capacity at 6 months with limited improvement beyond that limit. In addition, a significant prevalence of psychological impairments, such as post-traumatic stress disorder (39\%), depression (33\%), anxiety (30\%), and reduced quality of life was registered beyond 6 months [37].

\section{Strengths and weaknesses of the study}

The relevance of this study mainly relies on the prospective design and the extensive clinical, laboratory and instrumental evaluation of all patients.

However, there are also some limitations. First, the study was performed in a single center and included a limited number of patients. Second, it is not possible to know if the observed spirometric alterations were already present before SARS-CoV-2 infection. However, since only 9 per cent of our patients had chronic lung disease in their medical history, we can hypothesize that most of these changes are due to COVID-19. Third, in the acute phase of the disease, it was not possible to perform a spirometry both for logistical reasons and due to the high risk of instrument contamination. This prevented the possible comparison of such spirometric values with the values observed in other phases of the disease. Finally, this study analyzed the health status of COVID-19 patients after a relatively short period from discharge. A more extended follow-up is needed to better understand the possible long-term evolutions.

\section{Conclusions}

This study shows that a high percentage of COVID-19 patients continue to report symptoms 3-6 months after hospital discharge. Spirometry has shown frequent mild/ moderate alterations in DLCO, which were significantly associated with the persistence of exertional dyspnea and mild interstitial damage on thoracic US. Finally, laboratory tests have revealed an improvement in inflammatory and coagulation parameters and no significant late venous thrombotic complications has been observed. Our results support the need for early prevention and treatment of those symptoms of COVID-19 that may persist for a long time, to avoid or mitigate the clinical manifestations of "Long Covid". For this purpose, a multidisciplinary rehabilitation program, including physical, psychological, and psychiatric aspects of rehabilitation, has been proposed [26]. However, the effectiveness of these interventions has not been demonstrated yet and the results of ongoing clinical studies [38, 39] will be useful to define a better medical approach to the patient with "Long Covid".

Author contributions All authors contributed to the study conception and design. Material preparation and data collection were performed by all authors. Data analysis was performed by Alberto Fortini and Arianna Torrigiani. The first draft of the manuscript was written by Alberto Fortini and Arianna Torrigiani. All authors read and approved the final manuscript.

Funding This research did not receive any specific grant from funding agencies in the public, commercial, or not-for-profit sectors.

Availability of data and material All study data is stored in an electronic database (Microsoft Excel spreadsheet, version 2013) and is available for review.

\section{Declarations}

Conflicts of interest/Competing interests The authors declare that they have no conflicts of interest.

Ethics approval This study was approved by the Institutional Review Board of the Department of Medicine of the "Azienda USL Toscana Centro", Firenze, Italy. The authors declare that all procedures performed in this study are in accordance with ethical standards of the institutional and national research committee and with the 1964 Helsinki declaration and its later amendments or comparable ethical standards.

Consent to participate Patients provided written informed consent to participate in the study.

Consent for publication Patients signed informed consent regarding publishing their data.

\section{References}

1. Wu Z, McGoogan JM. Characteristics of and important lessons from the coronavirus disease 2019 (COVID-19) outbreak in China: summary of a Report of 72314 Cases From the Chinese Center for Disease Control and Prevention. JAMA. 2020;323:1239-42. https://doi.org/10.1001/jama.2020.2648 (PMID: 32091533). 
2. Chen N, Zhou M, Dong X, et al. Epidemiological and clinical characteristics of 99 cases of 2019 novel coronavirus pneumonia in Wuhan, China: a descriptive study. Lancet. 2020;395:507-13. https://doi.org/10.1016/S0140-6736(20)30211-7.

3. Huang C, Wang Y, Li X, et al. Clinical features of patients infected with 2019 novel coronavirus in Wuhan. China Lancet. 2020;395:497-506. https://doi.org/10.1016/S0140-6736(20) 30183-5.

4. Chen J, Qi T, Liu L, et al. Clinical progression of patients with COVID-19 in Shanghai. China J Infect. 2020;80:e1-6. https://doi. org/10.1016/j.jinf.2020.03.004

5. Temgoua MN, Endomba FT, Nkeck JR, Kenfack GU, Tochie JN, Essouma M. Coronavirus disease 2019 (COVID-19) as a multisystemic disease and its impact in low- and middle-income countries (LMICs). SN Compr Clin Med. 2020. https://doi.org/10. 1007/s42399-020-00417-7.

6. Chopra V, Flanders SA, O’Malley M, Malani AN, Prescott HC. Sixty- Day Outcomes Among Patients Hospitalized With COVID19. Ann Intern Med. 2020. https://doi.org/10.7326/M20-5661.

7. Spinicci M, Vellere I, Graziani L, et al. Clinical and laboratory follow-up after hospitalization for COVID-19 at an Italian tertiary care center. Open Forum Infect Dis. 2021;8:ofab049. https://doi. org/10.1093/ofid/ofab049.

8. Garrigues E, Janvier P, Kherabi Y, et al. Post-discharge persistent symptoms and health-related quality of life after hospitalization for COVID-19. J Infect. 2020;81:e4-6. https://doi.org/10.1016/j. jinf.2020.08.029.

9. Carfî A, Bernabei R, Landi F, Gemelli Against COVID-19 PostAcute Care Study Group. Persistent symptoms in patients after acute COVID-19. JAMA. 2020;324:603-5. https://doi.org/10. 1001/jama.2020.12603.

10. Huang C, Huang L, Wang Y, et al. 6-month consequences of COVID-19 in patients discharged from hospital: a cohort study. Lancet. 2021. https://doi.org/10.1016/S0140-6736(20)32656-8 (Epub ahead of print. PMID: 33428867).

11. Mo X, Jian W, Su Z, et al. Abnormal pulmonary function in COVID-19 patients at time of hospital discharge. Eur Respir J. 2020;55:2001217. https://doi.org/10.1183/13993003.01217-2020.

12. Salehi S, Reddy S, Gholamrezanezhad A. Long-term pulmonary consequences of coronavirus disease 2019 (COVID-19): what we know and what to expect. J Thorac Imaging. 2020;35:W87-9. https://doi.org/10.1097/RTI.0000000000000534.

13. Bosso G, Allegorico E, Pagano A, et al. Lung ultrasound as diagnostic tool for SARS-CoV-2 infection. Intern Emerg Med. 2020;3:1-6. https://doi.org/10.1007/s11739-020-02512-y.

14. Zuker-Herman R, Ayalon Dangur I, Berant R, Sitt EC, Baskin L, Shaya Y, Shiber S. Comparison between two-point and three-point compression ultrasound for the diagnosis of deep vein thrombosis. J Thromb Thrombolysis. 2018;45:99-105. https://doi.org/10.1007/ s11239-017-1595-9 (PMID: 29243193).

15. Hao F, Tan W, Jiang L, et al. Do psychiatric patients experience more psychiatric symptoms during COVID-19 pandemic and lockdown? A case-control study with service and research implications for immunopsychiatry. Brain Behav Immun. 2020;87:100 6. https://doi.org/10.1016/j.bbi.2020.04.069.

16. Bo HX, Li W, Yang Y, et al. Posttraumatic stress symptoms and attitude toward crisis mental health services among clinically stable patients with COVID-19 in China. Psychol Med. 2020;27:1-2. https://doi.org/10.1017/S0033291720000999.

17. Tng XJJ, Chew QH, Sim K. Psychological sequelae within different populations during the COVID-19 pandemic: a rapid review of extant evidence. Singap Med J. 2020. https://doi.org/10.11622/ smedj.2020111 (Epub ahead of print. PMID: 32729312).

18. Meini S, Suardi LR, Busoni M, Roberts AT, Fortini A. Olfactory and gustatory dysfunctions in 100 patients hospitalized for COVID-19: sex differences and recovery time in real-life. Eur
Arch Otorhinolaryngol. 2020;277:3519-23. https://doi.org/10. 1007/s00405-020-06102-8.

19. Li J, Long X, Zhu C, et al. Olfactory Dysfunction in Recovered Coronavirus Disease 2019 (COVID-19) Patients. Mov Disord. 2020;35:1100-1. https://doi.org/10.1002/mds.28172.

20. Boscolo-Rizzo P, Borsetto D, Fabbris C, et al. Evolution of altered sense of smell or taste in patients with mildly symptomatic COVID-19. JAMA Otolaryngol Head Neck Surg. 2020;146:729-32. https://doi.org/10.1001/jamaoto.2020.1379.

21. Desai SV, Law TJ, Needham DM. Long-term complications of critical care. Crit Care Med. 2011;39:371-9. https://doi.org/10. 1097/CCM.0b013e3181fd66e5 (PMID: 20959786).

22. Davydow DS, Giford JM, Desai SV, et al. Depression in general intensive care unit survivors: a systematic review. Intensive Care Med. 2009;35:796-809. https://doi.org/10.1007/ s00134-009-1396-5.

23. Boede M, Gensichen JS, Jackson JC, et al. Trajectories of depression in sepsis survivors: an observational cohort study. Crit Care. 2021;25:161. https://doi.org/10.1186/s13054-02103577-7 (PMID: 33926493).

24. Armange L, Bénézit F, Picard L, Pronier C, Guillot S, Lentz PA, Carré F, Tattevin P, Revest M. Prevalence and characteristics of persistent symptoms after non-severe COVID-19: a prospective cohort study. Eur J Clin Microbiol Infect Dis. 2021. https:// doi.org/10.1007/s10096-021-04261-y (Epub ahead of print. PMID: 33893570; PMCID: PMC8064701).

25. Jacobson KB, Rao M, Bonilla H, Subramanian A, Hack I, Madrigal $\mathrm{M}$, Singh U, Jagannathan $\mathrm{P}$, Grant P. Patients with uncomplicated COVID-19 have long-term persistent symptoms and functional impairment similar to patients with severe COVID19: a cautionary tale during a global pandemic. Clin Infect Dis. 2021. https://doi.org/10.1093/cid/ciab103 (Epub ahead of print. PMID: 33624010; PMCID: PMC7929039).

26. COVID-19 rapid guideline: managing the long-term effects of COVID-19. London: National Institute for Health and Care Excellence (UK); 2020 Dec 18. PMID: 33555768.

27. Marietta M, Coluccio V, Luppi M. COVID-19, coagulopathy and venous thromboembolism: more questions than answers. Intern Emerg Med. 2020;15:1375-87. https://doi.org/10.1007/ s11739-020-02432-x.

28. Fortini A, Beltrame C, Faraone A, Iandelli S, Zaccagnini G, Lo Forte A. Thromboinflammatory state and venous thromboembolic events in patients with coronavirus disease 2019 admitted to a nonintensive care unit: a prospective study. Pol Arch Intern Med. 2021;131:86-9. https://doi.org/10.20452/pamw.15625 (Epub 2020 Sep 25. PMID: 32975921).

29. Bourguignon A, Beaulieu C, Belkaid W, Desilets A, Blais $\mathrm{N}$. Incidence of thrombotic outcomes for patients hospitalized and discharged after COVID-19 infection. Thromb Res. 2020;196:491-3. https://doi.org/10.1016/j.thromres.2020.10. 017.

30. Allinovi M, Parise A, Giacalone M, et al. Lung ultrasound may support diagnosis and monitoring of COVID-19 pneumonia. Ultrasound Med Biol. 2020;46:2908-17. https://doi.org/10.1016/j. ultrasmedbio.2020.07.018

31. Bosso G, Vetrugno L, Bove T, et al. Our Italian experience using lung ultrasound for identification, grading and serial follow-up of severity of lung involvement for management of patients with COVID-19. Echocardiography. 2020;37:625-7. https://doi.org/10. 1111/echo.14664].

32. Tabatabaei SMH, Rajebi H, Moghaddas F, Ghasemiadl M, Talari H. Chest CT in COVID-19 pneumonia: what are the findings in mid-term follow-up? Emerg Radiol. 2020;27:711-9. https://doi. org/10.1007/s10140-020-01869-z.

33. Zhang Y, Xue H, Wang M, He N, Lv Z, Cui L. Lung ultrasound findings in patients with coronavirus disease (COVID-19). AJR 
Am J Roentgenol. 2021;216:80-4. https://doi.org/10.2214/AJR. 20.23513 (Epub 2020 Jul 22. PMID: 32755198).

34. Hui DS, Joynt GM, Wong KT, et al. Impact of severe acute respiratory syndrome (SARS) on pulmonary function, functional capacity and quality of life in a cohort of survivors. Thorax. 2005;60:401-9. https://doi.org/10.1136/thx.2004.030205.

35. Ngai JC, Ko FW, Ng SS, To KW, Tong M, Hui DS. The longterm impact of severe acute respiratory syndrome on pulmonary function, exercise capacity and health status. Respirology. 2010;15:543-50. https://doi.org/10.1111/j.1440-1843.2010. 01720.x.

36. Hui DS, Wong KT, Antonio GE, Tong M, Chan DP, Sung JJ. Long-term sequelae of SARS: physical, neuropsychiatric, and quality-of-life assessment. Hong Kong Med J. 2009;15:21-3.
37. Ahmed H, Patel K, Greenwood DC, et al. Long-term clinical outcomes in survivors of severe acute respiratory syndrome and Middle East respiratory syndrome coronavirus outbreaks after hospitalisation or ICU admission: a systematic review and metaanalysis. J Rehabil Med. 2020;52:jrm00063. https://doi.org/10. 2340/16501977-2694.

38. Rutsch M, Frommhold J, Buhr-Schinner H, Djeiranachvili L, Gross T, Schüller PO, Katalinic A, Deck R. Study protocol medical rehabilitation after COVID-19 disease: an observational study with a comparison group with obstructive airway disease / Re_Co. BMC Health Serv Res. 2021;21:373. https://doi.org/10.1186/ s12913-021-06378-4 (PMID:33888104;PMCID:PMC8061875).

39. The Post-hospitalisation COVID-19 Study (PHOSP-COVID). https://www.phosp.org. Accessed May 10, 2021. 